\title{
Plant Leaf Recognition through Local Discriminative Tangent Space Alignment
}

\author{
Chuanlei Zhang, ${ }^{1}$ Shanwen Zhang, ${ }^{2}$ and Weidong Fang ${ }^{3}$ \\ ${ }^{1}$ School of Science and Information Engineering, Tianjin University of Science and Technology, Tianjin 300222, China \\ ${ }^{2}$ Department of Electronics and Information Engineering, Xijing University, Xian 710123, China \\ ${ }^{3}$ Key Laboratory of Specialty Fiber Optics and Optical Access Networks, Shanghai University, Shanghai 200444, China
}

Correspondence should be addressed to Shanwen Zhang; wjdw716@163.com

Received 5 December 2015; Accepted 15 February 2016

Academic Editor: Hui Cheng

Copyright (C) 2016 Chuanlei Zhang et al. This is an open access article distributed under the Creative Commons Attribution License, which permits unrestricted use, distribution, and reproduction in any medium, provided the original work is properly cited.

\begin{abstract}
Manifold learning based dimensionality reduction algorithms have been payed much attention in plant leaf recognition as the algorithms can select a subset of effective and efficient discriminative features in the leaf images. In this paper, a dimensionality reduction method based on local discriminative tangent space alignment (LDTSA) is introduced for plant leaf recognition based on leaf images. The proposed method can embrace part optimization and whole alignment and encapsulate the geometric and discriminative information into a local patch. The experiments on two plant leaf databases, ICL and Swedish plant leaf datasets, demonstrate the effectiveness and feasibility of the proposed method.
\end{abstract}

\section{Introduction}

Plant recognition based on leaf images plays an important role in agricultural informatization, ecological protection, and automatic plant recognition system. One of the most important steps in the image based plant recognition is to validly extract classifying features. Currently, the commonly employed classifying features for plant recognition based on leaf image could be categorized into color, shape, and texture features [1-3]. Plant leaf classification is a challenging problem due to its high dimensionality data, complexity, and irregular shape of plant leaf images [4-6]. Traditional dimensionality reduction methods typically have a smaller data space from linear combinations of the original data. The most common example is principal component analysis (PCA), which seeks a low-dimensional linear subspace spanned by the eigenvectors which correspond to the largest eigenvalues of the covariance matrix of all the samples. However, for plant leaf images, the assumption of global linearity is a severe constraint because the leaf images are quite sensitive to seasonality, location, and illumination conditions. Thus, it is not reasonable to believe that the leaf image data could be linearly separable from each other.
Manifold learning has been utilized in many applications such as pattern recognition, visualization, and classification tasks. In the last ten years, many manifold learning nonlinear algorithms have been introduced with an assumption that the processed data lies on or close to some low-dimensional manifolds which are embedded in a high-dimensional unorganized Euclidean space. In these manifold learning algorithms, the most representative ones are isometric feature mapping (ISOMAP) in [7], locally linear embedding (LLE) in [8], Laplacian eigenmaps (LE) in [9], Hessian-based locally linear embedding (HLLE) in [10], maximum variance unfolding (MVU) in [11], local tangent space alignment (LTSA) in [12], local spline embedding (LSE) in [13], and local discriminative tangent space alignment (LDTSA) in [14]. One of the most important advantages of manifold learning [7-14] compared with conventional methods is how the data are treated mathematically. Manifold learning methods allow the data to be related nonlinearly, which leads to the fact that manifold learning methods can much more accurately capture the proper structures among the data, thus allowing for accurate recognition. For every manifold learning algorithm, it tries to preserve a different geometrical property of the underlying manifold. Local methods such as LLE, HLLE, LE, LTSA, 
and LSE try to preserve the neighborhood structure in the data, while global methods like ISOMAP aim to preserve the metrics at all scales. Thanks to their nonlinear nature, geometric intuition, and computational feasibility, these nonlinear methods have promising results on some artificial and real-world datasets. In [15] a framework, which is called patch alignment, was proposed and it consists of two stages: part optimization and whole alignment. In this paper, we take an alternative view of the framework to introduce an efficient method based on local discriminative tangent space alignment (LDTSA) for plant leaf recognition. Compared with current plant leaf recognition methods, the proposed one can avoid the small sample size problem, preserves the discriminative capability, and detects the intrinsic structure from the plant leaf image data.

The paper is organized as follows: Section 2 briefly describes the dimensionality reduction algorithm based on local discriminative tangent space alignment and its procedures. Experiments on plant leaf database are offered in Section 3 and the paper is ended with some conclusions in Section 4.

\section{Local Discriminative Tangent Space Alignment Algorithm}

Suppose $n$ original labeled data points $X=\left[x_{1}, \ldots, x_{n}\right]$, including all the samples $x_{i} \in R^{m}, i=1,2, \ldots, n$. The objective of a dimensionality reduction algorithm is to compute the corresponding low-dimensional representations of $X Y=\left[y_{1}, \ldots, y_{n}\right], y_{i} \in R^{d}, i=1,2, \ldots, n$, where $d \ll$ $m$. For the linear dimensionality reduction, it is necessary to find projection matrix $A$, such that $Y=A^{T} X$. For the nonlinear dimensionality reduction, it is usually difficult to provide an explicit mapping to transform data from a high-dimensional space to a low-dimensional subspace. For classification task, in part optimization stage we always hope to project the high-dimensional data into a low-dimensional feature space, in which the projection is characterized by within-class compactness and between-class separability [15]. Assume that there is an interaction force between any pairwise points in the ambient space; the mutual force can be distinguished as within-class attraction or between-class repulsion between any pairwise points from the same or different class, respectively (see Figure 1) [16].

In the reduced subspace, in order to achieve within-class attraction for data point $y_{i}$, the following objective function is defined as

$$
\arg \min \sum_{j=1}^{k_{1}}\left\|y_{i}-y_{i}\right\|^{2}
$$

where $k_{1}$ is the number of the nearest neighbors with respect to $x_{i}$ from data points in the same class as $x_{i}$.

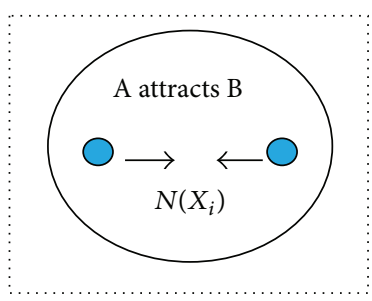

(a)

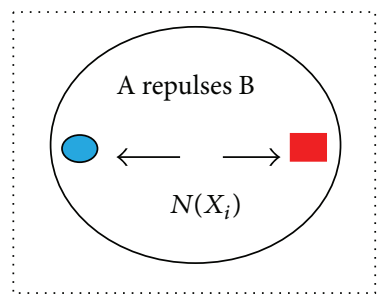

(b)
FIGURE 1: An intuitive demonstration of within-class attraction and between-class repulsion between pairwise points, where the circle denotes $k$ nearest neighbors $N\left(X_{i}\right)$ of $X_{i}$; (a) A and B belong to the same class; (b) A and B belong to different classes.

In order to achieve between-class separability purpose for the data point $y_{i}$, the following objective function is defined as

$$
\arg \max \sum_{p-1}^{k_{2}}\left\|y_{i}-y_{i_{p}}\right\|^{2}
$$

LTSA uses tangent coordinates to indicate the local geometry. Assume that there is an affine projection matrix, which projects tangent coordinates to the low-dimensional coordinates in a local patch which contains neighbors from both the same and different classes. To obtain the optimal tangent coordinates, we have the following objective function on each patch:

$$
\arg \min \left\|Y_{i} R_{k+1}-T_{i} \Theta_{i}\right\|^{2},
$$

where $R_{k+1}=I_{k+1}-e_{k+1} e_{k+1}^{T} /(k+1)$ denotes the centralization matrix; $e_{k+1}=[1, \ldots, 1]^{T} \in R^{k+1} ; I_{k+1}$ is $(k+1) \times(k+1)$ identity matrix; and $\Theta_{i} \in R^{d \lambda(k+1)}$ is the tangent coordinates corresponding to an orthonormal basis matrix of the tangent space.

Since the patch formed by the local neighborhood can be considered approximately linear, we write the part discriminator by using the linear manipulation as follows:

$$
\begin{aligned}
& \underset{y_{i}}{\operatorname{argmin}}\left(\sum_{j-1}^{k_{i}}\left\|y_{i}-y_{i^{j}}\right\|^{2}\right. \\
& \left.-\alpha \sum_{p-1}^{k_{2}}\left\|y_{i}-y_{i_{p}}\right\|^{2}+\beta\left\|Y_{i} R_{k+1}-T_{i} \Theta_{i}\right\|^{2}\right),
\end{aligned}
$$

where $\alpha$ and $\beta$ are scaling factors to unify different data points of the within-class distance and the between-class distance and they are selected based on experiments.

Then objective function (4) can be rewritten by patch alignment:

$$
\begin{aligned}
& \underset{Y_{i}}{\operatorname{argmin}} \sum_{j=1}^{l}\left[\operatorname{tr}\left(Y_{i} L_{i_{w 1}} Y_{i}^{T}\right)+\beta \operatorname{tr}\left(Y_{i} L_{i_{w 2}} Y_{i}^{T}\right)\right. \\
& \left.\quad-\alpha \operatorname{tr}\left(Y_{i} L_{i_{b}} Y_{i}^{T}\right)\right],
\end{aligned}
$$


where

$$
\begin{aligned}
L_{i_{w 1}} & =\left[\begin{array}{cc}
\sum_{j=1}^{k_{1}}\left(\omega_{i}\right)_{j} & -\omega_{i}^{T} \\
-\omega_{i} & \operatorname{diag}\left(\omega_{i}\right)
\end{array}\right], \\
L_{i_{w 2}} & =R_{k_{1}+1}-V_{i} V_{i}^{T}, \\
L_{i_{b}} & =\left[\begin{array}{cc}
\sum_{j=1}^{k_{2}}\left(\omega_{i}\right)_{j} & -\omega_{i}^{T} \\
-\omega_{i} & \operatorname{diag}\left(\omega_{i}\right)
\end{array}\right],
\end{aligned}
$$

where $V_{i}$ denotes the matrix of $d$ right singular vectors of $X_{i} R_{k+1}$ corresponding to its $d$ largest values; and $\omega=[\overbrace{1, \ldots, 1}^{k_{1}}$ $\overbrace{-\beta, \ldots,-\beta}^{k_{2}}]^{T}$ is a coefficient vector.

In (5), the first two parts only involve the data points belonging to within-class neighbors and they share the same selection matrix $S_{w}$. The third part concerns the betweenclass neighbors and uses selection matrix $S_{b}$.

Then (5) can be reformed to the following:

$$
\begin{aligned}
& \underset{Y_{i}}{\operatorname{argmin}} \sum_{i-1}^{l}\left[\operatorname{tr}\left(Y_{L} S_{w} L_{i_{w}}\left(Y_{L} S_{w}\right)^{T}\right)+\alpha \operatorname{tr}\left(Y_{L} S_{b}\right)^{T}\right] \\
& \quad=\underset{Y_{i}}{\arg \min } \operatorname{tr}\left(Y_{L} L Y_{L}^{T}\right),
\end{aligned}
$$

where $S_{w}$ and $S_{b}$ are selection matrix and

$$
\begin{aligned}
L_{i_{w}} & =L_{i_{w 1}}+\beta L_{i_{w 2}}, \\
L & =\sum_{i=1}^{l}\left(S_{w} L_{i_{w}} S_{w}^{T}+\alpha S_{b} L_{b} S_{b}^{T}\right) .
\end{aligned}
$$

In summary, the main procedure of the proposed algorithm for the plant leaf image classification task can be described as follows.

Step 1. Select representative labeled plant leaf image samples to which the following dimensionality reduction will be done.

Step 2. For each point $x_{i}$, find its within-class neighborhood set $N_{w}\left(x_{i}\right)$ with $k_{1}$ elements and between-class neighborhood set $N_{w}\left(x_{i}\right)$ with $k_{2}$ elements.

Step 3. Generate two vectors $X_{w}=\left[x_{i_{1}}, \ldots, x_{i_{k_{1}}}\right]$ and $X_{b}=$ $\left[x_{i_{1}}, \ldots, x_{i_{k_{2}}}\right]$, with their elements from $N_{w}\left(x_{i}\right)$ and $N_{w}\left(x_{i}\right)$, respectively.

Step 4. Construct $L_{i_{w 1}}$ with $k_{1}$ within-class neighbors, then centralize the neighbors and compute the top- $d$ eigenvector from the autocorrection matrix, and then construct $L_{i_{w 2}}$ and record the selection matrix $S_{w}$.

Step 5. Construct $L_{i_{b}}$ with $k_{2}$ between-class neighbors, and record the selection matrix $S_{b}$.

\section{0}

(a)

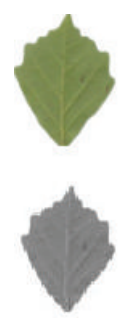

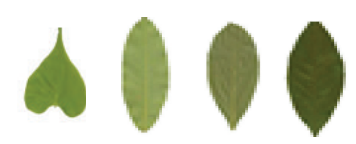

(b)
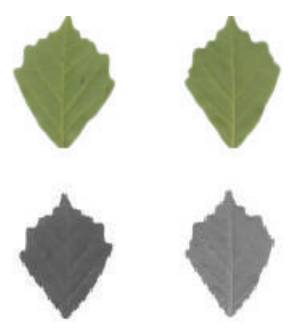

(c)
Step 6. Generate global $L$ with patch alignment through respective selection matrix.

Step 7. Perform eigendecomposition on $X L X^{T}$ and the eigenvectors form projection matrix $U$.

Step 8. Final reduced dimensionality results are $Y_{L}=U^{T} X$.

\section{Experiment Results}

3.1. Experiment Results on ICL Dataset. ICL leaf database has 17032 plant leaf images of 220 species and image number of each class is unequal [17]. In order to verify the effectiveness of the proposed method in this paper, we construct one leaf image subset from the ICL leaf dataset, which has 15 species with 11 samples per species, and all classes are carefully chosen so that the shapes could be distinguished easily by human eyes or the shapes are similar but still can be identified [16]. Some typical example images are demonstrated in Figures 2(a), 2(b), and 2(c). Preprocessing is performed to crop all leaf images from two databases. The illuminations keep the same condition and the backgrounds are white, and the size of each cropped leaf image in experiments is $64 \times 64$ pixels, with gray level of 256 gray levels per pixel in preprocessing step, as demonstrated in Figure 2(c) of one species.

Then, every image is represented in a 4096-dimensional vector in the image space. By prereducing by PCA, 98 percent image energy is kept and all principal components are selected corresponding to the nonzero eigenvalues for each method. The 1-NN classifier is employed to classify leaf images for its simplicity. The distance measure is Euclidean distance.

The leaf image dataset is randomly separated into two subsets: one part is for training (sizes are 30, 45, 60, 75, 105, and 120) and the other is for testing purpose. The training sets are used to obtain the low-dimensional subspace with a projection matrix. The testing sets are utilized to test the final 
TABLE 1: Average classification rates (\%) and standard deviations ICL plant leaf database.

\begin{tabular}{lccc}
\hline Train samples & LSDA & LLTSA & LDTSA \\
\hline 30 & $86.26 \pm 3.52$ & $73.3 \pm 3.48$ & $86.56 \pm 3.62$ \\
45 & $88.83 \pm 3.63$ & $78.33 \pm 3.39$ & $89.33 \pm 3.04$ \\
60 & $91.81 \pm 2.29$ & $82.19 \pm 2.22$ & $92.71 \pm 2.38$ \\
75 & $91.61 \pm 2.76$ & $81.39 \pm 2.56$ & $92.93 \pm 2.63$ \\
105 & $93.25 \pm 2.62$ & $82.83 \pm 2.76$ & $94.08 \pm 2.78$ \\
120 & $94.12 \pm 2.54$ & $80.67 \pm 3.26$ & $94.56 \pm 3.78$ \\
\hline
\end{tabular}

TABLE 2: Average classification rates (\%) and standard deviations Swedish plant leaf database.

\begin{tabular}{lccc}
\hline Train samples & LSDA & LLTSA & LDTSA \\
\hline 300 & $82.35 \pm 3.45$ & $71.3 \pm 3.62$ & $84.59 \pm 3.34$ \\
600 & $84.73 \pm 3.58$ & $75.39 \pm 3.76$ & $87.38 \pm 3.21$ \\
900 & $89.67 \pm 3.31$ & $80.49 \pm 2.89$ & $91.91 \pm 2.73$ \\
\hline
\end{tabular}

classification accuracy. Each time the test is repeated 20 times and the accuracy rate is calculated each time, as follows:

$$
\text { Accuracy }=\frac{\operatorname{Num}(R)}{\operatorname{Num}(T)} \cdot 100 \%,
$$

where $\operatorname{Num}(R)$ is the right sample number detected and $\operatorname{Num}(T)$ is the total sample number tested.

Table 1 shows the average classification rates and standard deviations of three algorithms in our experiments on the selected datasets which are locality sensitive discriminant analysis (LSDA), linear local tangent space alignment (LLTSA), and the proposed LDTSA. It can be seen that the proposed method obtains better accuracy.

3.2. Experiment Results on Swedish Dataset. Swedish leaf dataset [18] has 1125 images from 15 different plant species, with 75 leaf images per species. The preprocess of the leaf image is the same as ICL dataset [16]. For each method, random subsets with 20,40 , and 60 images per species are selected for training, the rest for testing. Such experiment with a specific number is independently performed 20 times, and then the best average classification results are recoded. Table 2 shows the maximal average classification accuracy with different size of training sets and test sets. It could be found that the proposed method outperforms the other algorithms in all the cases.

\section{Conclusions}

Plant recognition based on leaf images has been an important and difficult research topic, especially for leaves with different and complicated shapes. Although there are many existing algorithms for plant leaf recognition, the recognition rates are still low due to the complexity of plant leaf. Manifold learning based dimensionality reduction algorithms are promising alternatives to traditional plant leaf recognition methods. A dimensionality reduction method based on local discriminative tangent space alignment (LDTSA) is proposed for plant leaf recognition task in this paper, and it embraces part optimization and whole alignment and encapsulates the geometric and discriminative information into a local patch. The experiment performed on two plant leaf databases shows the effectiveness and feasibility of the proposed method in this paper.

\section{Competing Interests}

The authors declare that there is no conflict of interests regarding the publication of this paper.

\section{Acknowledgments}

The paper was supported by Tianjin Research Program of Application Foundation and Advanced Technology 14JCYBJC42500 and Tianjin City High School Science \& Technology Fund Planning Project 20140802. It was partly funded by the young academic team construction projects of the "twelve five" integrated investment planning in Tianjin University of Science and Technology and the 2015 key projects of Tianjin Science and Technology Support Program no. 15ZCZDGX00200. This work was also supported by the National Natural Science Foundation of China under Grant no. 61502338 and the Open Fund of Guangdong Provincial Key Laboratory of Petrochemical Equipment Fault Diagnosis no. GDUPTKLAB201334.

\section{References}

[1] A. El-ghazal, O. A. Basir, and S. Belkasim, "Shape-based image retrieval using pair-wise candidate co-ranking," in Image Analysis and Recognition: 4th International Conference, ICIAR 2007, Montreal, Canada, August 22-24, 2007. Proceedings, M. S. Kamel and A. C. Campilho, Eds., vol. 4633 of Lecture Notes in Computer Science, pp. 650-661, Springer, Berlin, Germany, 2007.

[2] S. Zhang and Y.-K. Lei, "Modified locally linear discriminant embedding for plant leaf recognition," Neurocomputing, vol. 74, no. 14-15, pp. 2284-2290, 2011.

[3] J. Han and K.-K. Ma, "Rotation-invariant and scale-invariant Gabor features for texture image retrieval," Image and Vision Computing, vol. 25, no. 9, pp. 1474-1481, 2007.

[4] F. Mokhtarian and S. Abbasi, "Matching shapes with selfintersections: application to leaf classification," IEEE Transactions on Image Processing, vol. 13, no. 5, pp. 653-661, 2004.

[5] Y. F. Li, Q. S. Zhu, Y. K. Cao, and C. L. Wang, "A leaf vein extraction method based on snakes technique," in Proceedings of IEEE International Conference on Neural Networks and Brain (ICNN\&B '05), pp. 885-888, Beijing, China, October 2005.

[6] S.-W. Zhang and C.-L. Zhang, "Two-dimensional locality discriminant projection for plant leaf classification," Intelligent Computing Theories and Applications Lecture Notes in Computer Science, vol. 7390, pp. 82-88, 2012.

[7] J. B. Tenenbaum, V. de Silva, and J. C. Langford, "A global geometric framework for nonlinear dimensionality reduction," Science, vol. 290, no. 5500, pp. 2319-2323, 2000.

[8] S. T. Roweis and L. K. Saul, "Nonlinear dimensionality reduction by locally linear embedding," Science, vol. 290, no. 5500, pp. 2323-2326, 2000. 
[9] M. Belkin and P. Niyogi, "Laplacian eigenmaps for dimensionality reduction and data representation," Neural Computation, vol. 15, no. 6, pp. 1373-1396, 2003.

[10] D. L. Donoho and C. Grimes, "Hessian eigenmaps: locally linear embedding techniques for high-dimensional data," Proceedings of the National Academy of Sciences of the United States of America, vol. 100, no. 10, pp. 5591-5596, 2003.

[11] K. Q. Weinberger and L. K. Saul, "Unsupervised learning of image manifolds by semidefinite programming," in Proceedings of the IEEE Computer Society Conference on Computer Vision and Pattern Recognition (CVPR '04), vol. 2, pp. II-988-II-995, IEEE, June-July 2004.

[12] Z. Zhang and H. Zha, "Principal manifolds and nonlinear dimensionality reduction via tangent space alignment," SIAM Journal on Scientific Computing, vol. 26, no. 1, pp. 313-338, 2005.

[13] S. M. Xiang, F. P. Nie, C. S. Zhang, and C. Zhang, "Nonlinear dimensionality reduction with local spline embedding," IEEE Transactions on Knowledge and Data Engineering, vol. 21, no. 9, pp. 1285-1298, 2009.

[14] Q. Shi, B. Du, and L. Zhang, "A dimensionality reduction method for hyperspectral imagery based on local discriminative tangent space alignment," Acta Geodaetica et Cartographica Sinica, vol. 41, no. 3, pp. 417-420, 2012.

[15] T. Zhang, D. Tao, X. Li, and J. Yang, "Patch alignment for dimensionality reduction," IEEE Transactions on Knowledge and Data Engineering, vol. 21, no. 9, pp. 1299-1313, 2009.

[16] S. Zhang, Y. Lei, T. Dong, and X.-P. Zhang, "Label propagation based supervised locality projection analysis for plant leaf classification," Pattern Recognition, vol. 46, no. 7, pp. 1891-1897, 2013.

[17] Intelligent Computing Laboratory Plant Leaf Dataset, http:// www.intelengine.cn/English/dataset/index.html.

[18] Söderkvist, Computer vision classification of leaf from Swedish trees [M.S. thesis], Linköping University, Linköping, Sweden, 2001. 


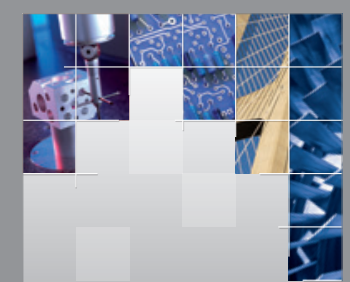

\section{Enfincering}
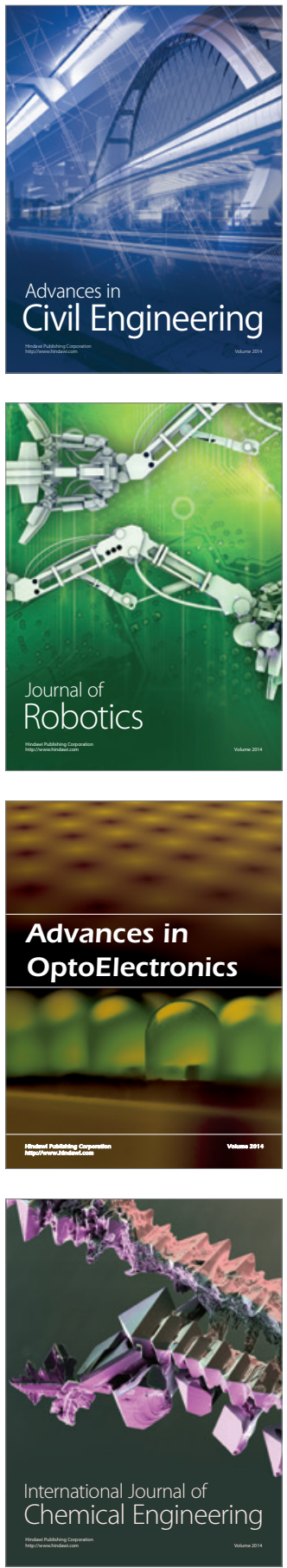

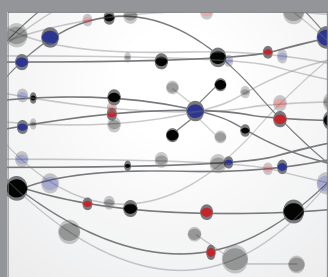

The Scientific World Journal

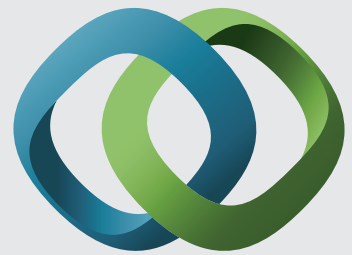

\section{Hindawi}

Submit your manuscripts at

http://www.hindawi.com
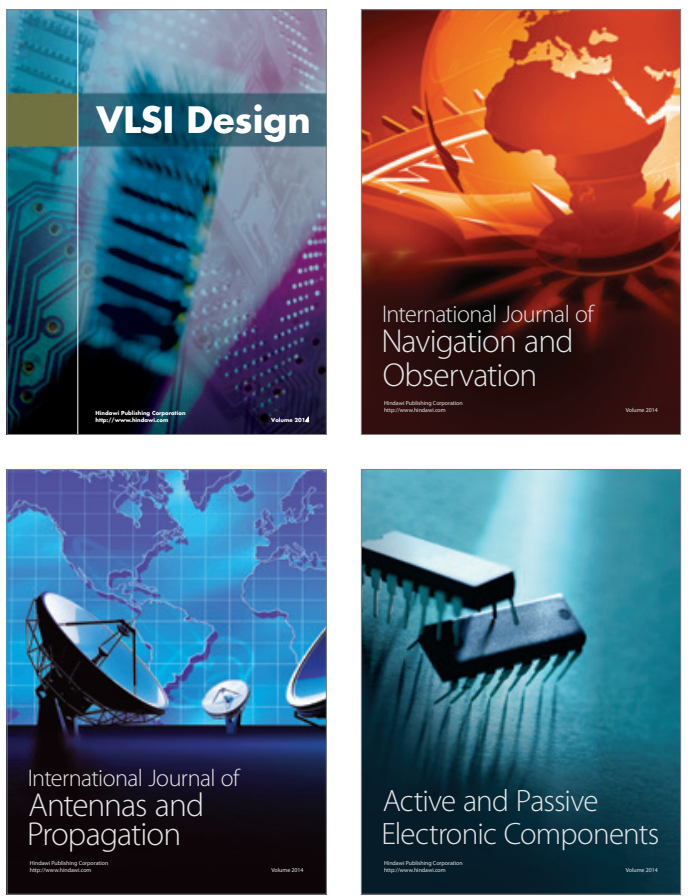
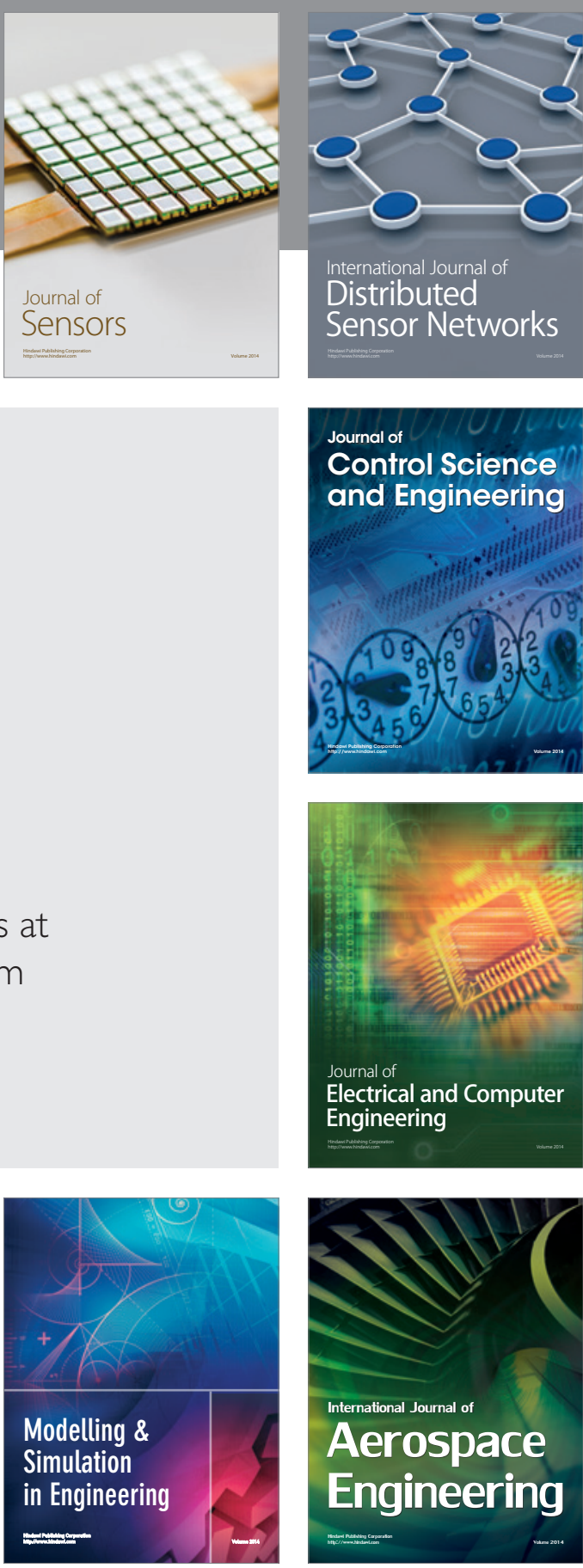

International Journal of

Distributed

Sensor Networks

Journal of

Control Science

and Engineering
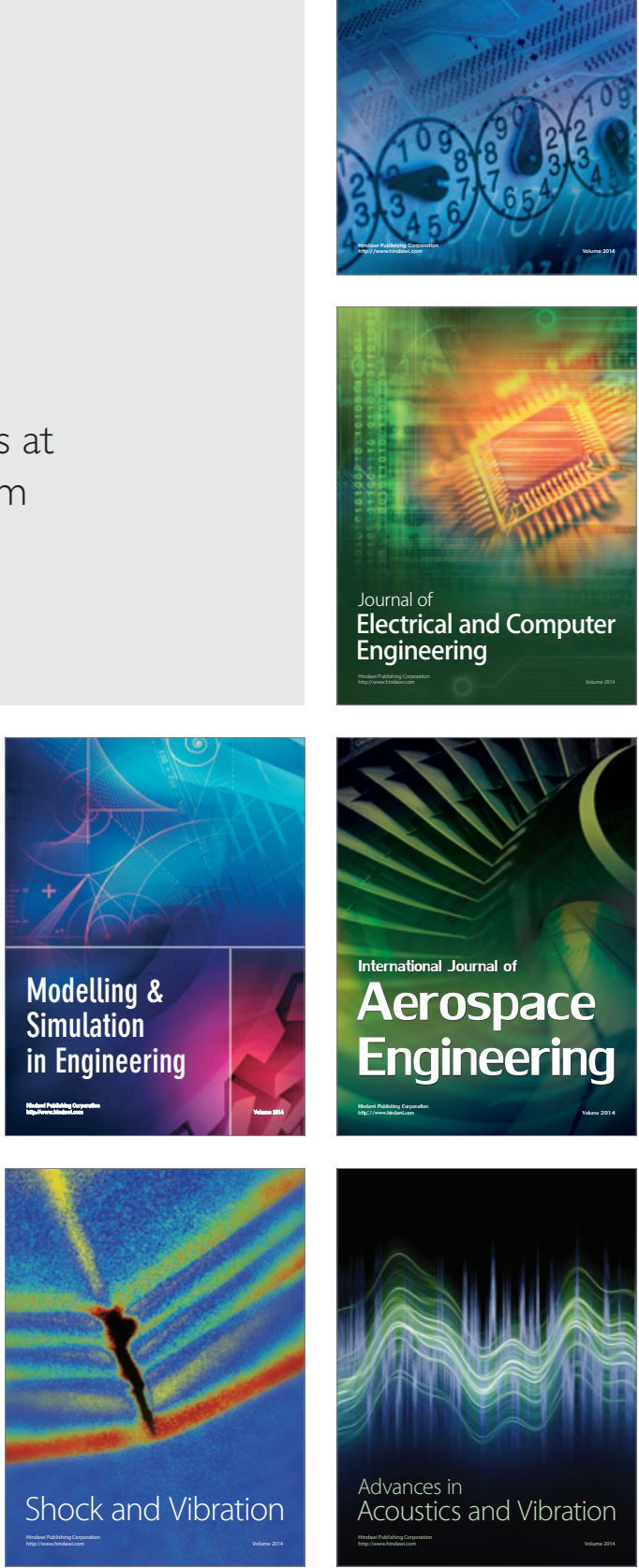